\title{
A Weak Theory of Strong Readings
}

\author{
Daniel Büring \\ Cologne University
}

\section{Introduction}

As is well-known, Focus can affect the meaning of sentences involving adnominal quantifiers in various ways. This paper deals with three of the cases discussed in the literature, which I refer to as strong or partitive readings of weak quantifiers ((1)), proportional readings of weak and strong quantifiers ((2)), and focus-affected readings of weak quantifiers ((3); capitals indicate stress).

(1) SOME cowboys decided to go HOME.

$\approx$ some of the cowboys decided to go home

(2) a. MOST boys WALKED to the station.

$\approx$ most boys who somehow got to the station walked there

b. THREE boys WALKED to the station.

$\approx$ three of the boys who somehow got to the station walked to the station

(3) Few/FEW INCOMPETENT cooks applied.

$\approx$ few of the applying cooks were incompetent

Below the examples I have given paraphrases of the readings we are concerned with here, and which seem the most natural ones for these examples. To see that these are remarkable, let us briefly recapitulate what the interpretation of a wellbehaved adnominal quantifier should look like. Syntactically a quantifier like some in (1) has as its sister a nominal argument, which - for lack of commitment - I refer to as the $N$-argument. The resulting NP (DP) is sister to a verbal projection, labeled the $V$-argument in the remainder of this paper. Ideally, we would like the meaning of $\mathrm{N}$ to provide the first argument - the restrictor - of the quantifier, and $\mathrm{V}$ to provide the second - the nuclear scope - in the semantics. An ideal mapping would thus be as in (4).

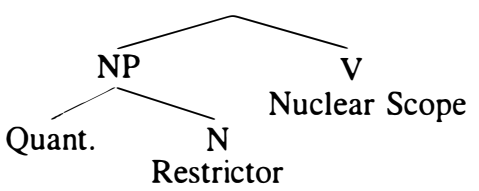

Against this background, the paraphrases in (1) through (3) are indeed weird: In (1) the partitive marker of the seems to appear out of thin air. In (2) material from the V-argument figures in the restrictor (paraphrased by the relative clauses), and finally, in (3) part of the $\mathrm{V}$ argument serves as the restrictor, while part of the $\mathrm{N}$ argument shows up in the nuclear scope.

As said above, all of these phenomena have received concise syntactic and semantic treatments in order to account for their peculiarities (e.g. Eckardt 1994, 
Geilfuß 1993, Herburger 1992, de Hoop 1992, de Hoop \& Solà 1995, Jäger 1995, Partee 1989). Most of these theories have in common that they adjust the compositional semantics (and some of them the syntax) in such a way that the truth-conditions of the sentences actually change (usually through some rule of association with focus). Let us call these semantic accounts.

In this paper I will elaborate on these accounts, making three claims: i) all these phenomena are instances of the same general phenomenon, ii) the appropriate treatment of these examples is pragmatic, and iii) no construction specific rules or devices are necessary (i.e. special rules of LF construal, lexical ambiguities of quantifiers, and/or rules of association with focus that are specific to certain constructions or lexical items). I thus advocate a pragmatic account.

Accordingly, the paper consist of two parts: A) to show that such an alternative and simpler approach can be provided, and B) to demonstrate that it is empirically superior to the analyses referred to above. As for A), there are two basic ingredients to the pragmatic account: For one thing, strong adnominal quantifiers are restricted by Westerståhlian Resource Domain Variables (RDVs). For another, the process of selecting RDVs in out-of-the-blue cases heavily relies on Topic/Focus/Background structure (TFBS). The particular version of Focus theory used is an extended Roothian focus semantics as proposed in Büring 1995.

The upshot of the B)-part will be that the truth conditions sketched in the paraphrases above and derived by the compositional semantics in the semantic accounts are mostly too strict, capturing only a subset of the possible readings of these sentences, namely the out-of-context cases. In providing specific contexts I will show that the sentences can convey meanings that are incompatible with the truth conditions of the semantic accounts, but predicted by the pragmatic one. This is why I refer to the latter as a 'weak' analysis: It assigns truth conditions that are much less specific than in the semantic accounts.

I will first outline the assumptions about TFBS I am making (section 1) and then turn to the analysis of the weak quantifier cases (section 2). Following that I will introduce the concept of RDVs and apply them in the analyses of the strong quantifier cases (section 3). Finally I'll turn to focus-affected readings (section 4). In comparing the present proposal to the ones made in the literature in terms of truth conditions I will use a somewhat unified version of the latter, basically to facilitate comparison. I therefore include an appendix where some of the proposed analyses are summarized and compared in more detail.

\section{Guessing Contexts: Topic/Focus/Background Structure}

One peculiarity of the sentences in (1) through (3) is that none of them can normally be used as a discourse initial sentence. For one thing, adnominal quantifiers like the given ones require aforementioned groups or domains. For another, the TFBS is incompatible with sentence Focus, that is, none of the sentences with the accentuation indicated can represent an all-new utterance. Assuming that discourse initial sentences need to be all-new, (1) through (3) call for specified contexts. 
What do we do when we encounter such a sentence out of the blue? Naturally enough, we try to construct a context by means of whatever information the pertinent sentence itself provides. And the best source of information we have - apart from lexically based presuppositions - is the TFBS, as indicated by intonation. Let us therefore look at the relation between intonation and context in more detail.

\subsection{Focus}

According to standard wisdom, Focus (indicated by a falling pitch contour) signals the position of new information in the sentence. In question/answer pairs, the focused constituent must correspond to the $w h$-phrase in the question, i.e. the requested information (the actual Focus - in contradistinction to the accent - is marked by a subscript F, as proposed in Jackendoff 1972).

(5) a. What did she give to John?

b. She gave [the $\mathrm{BOOK}]_{\mathrm{F}}$ to John.

b. \# She GAVE $\mathrm{F}_{\mathrm{F}}$ the book to John.

We take asking questions to be an archetypal case of establishing a Discourse Topic (or D-Topic). Questions, and D-Topics in general are represented as sets of propositions, namely the set of possible answers/possible continuations of the discourse (Hamblin 1973). The term question will be used throughout for sets of propositions (suggesting that every declarative answers an (implicit) question).

Since we know that the Focus in the answer must correspond to the $w h$-part of the question, we can say that a declarative sentence with Focus defines the (set of) question(s) it is suited to answer. Descriptively the question is derived by replacing the focus by an appropriate wh-word (ignoring yes/no questions). Formally, the question is the set of propositions we get by replacing the meaning of the Focus in the sentence meaning with (contextually salient and plausible) type identical alternatives. Following Rooth 1985 we call this set of propositions the Focus Value of sentence $S$, $\llbracket S \rrbracket^{\mathrm{f}}$. Question/answer matching then is nothing more than the requirement that $\llbracket S \rrbracket^{\mathrm{f}}$ equal the D-Topic.

(6) Discourse Appropriateness Condition (preliminary):

For a question/answer pair Q/A to be wellformed, it must hold that $\llbracket Q \rrbracket^{\circ}=\llbracket A \rrbracket^{f}{ }^{1}$

Upon hearing a sentence $S$ out-of-the-blue it is a good guess to take the D-Topic to be $\llbracket S \rrbracket^{f}$. We refer to this as reconstructed D-Topics or reconstructed questions (henceforth I will use paraphrases in a $\lambda$-categorial language as in (7.b) to characterize such meanings).

(7) a. She gave [the $\mathrm{BOOK}]_{\mathrm{F}}$ to John.

b. 【she gave [the BOOK $]_{\mathrm{F}}$ to John $\rrbracket^{f}=\{\llbracket$ she gave the book to John $\rrbracket^{\circ}$, 【 she gave the journal to John $\rrbracket^{\circ}$, 【 she gave the newspaper to John $\left.\rrbracket^{\circ}, \ldots\right\}=\llbracket \lambda p . \exists x \in A L T$ (the-book) \& $\mathrm{p}=$ she gave $\mathrm{x}$ to John $\rrbracket^{\circ}=\llbracket$ What did she give to John? $\rrbracket^{\circ}$ 
We pretend that a declarative sentence with focus allows for reconstructing the D-Topic.

\subsection{Topic}

Next note that the sentences we are discussing involve two accents, the first of which is not a Focus accent, but what I call a Topic accent. A constituent thus marked is called a Sentence Internal Topic or S-Topic - indicated by $[\ldots]_{\mathrm{T}}$ brackets. To see why S-Topics are different from Foci let us look at their impact on question/answer pairs. Unlike the Focus, S-Topics do not correspond to the $w h$-word. Rather they indicate a departure from the original question. Consider (8):

(8) What did the older boys do?

a. The older boys [ate ICE CREAM] $]_{\mathrm{F}}$.

b. $[\mathrm{BILL}]_{\mathrm{T}}$ [ate ICE CREAM $]_{\mathrm{F}}$.

c. The [YOUNG] $]_{\mathrm{T}}$ boys [ate ICE CREAM] $]_{\mathrm{F}}$.

d. The [GIRLS $]_{\mathrm{T}}$ [ate ICE CREAM $]_{\mathrm{F}}$.

e. \# Bill [ate ICE CREAM] $]_{F}$.

f. \# The young boys [ate ICE CREAM] $]_{F}$.

g. \# The girls [ate ICE CREAM] $]_{\mathrm{F}}$.

(8.a) presents the 'ordinary' answer with just a Focus accent. The answers in (8.b) through (8.d), however, do not match the question (formally: $\llbracket A \rrbracket^{\mathrm{f}} \neq$ $\llbracket Q \rrbracket^{\circ}$, as can easily be seen by just replacing the Focus with the appropriate question word). To legitimate such a deviance, an S-Topic has to be present on the constituent where D-Topic and $\llbracket A \rrbracket^{\mathrm{f}}$ differ. Note that leaving out the first accent, as shown in (8.e) through (8.g), renders these sentences illformed as answers to the question in (8), just as we would expect given (6).

$\mathrm{S}$-Topics are thus used if the Focus Value of A does not equal the D-Topic. This effect can be described as follows: Replacing the meaning of the S-Topic by an appropriate type identical alternative must yield the D-Topic (the older boys, older and older boys, respectively, in (8.b) through (8.d)).

Hearing a declarative sentence with S-Topic and Focus out-of-the-blue we know that one of the questions we can get by i) replacing the focus by the corresponding wh-word, and ii) replacing the $S$-Topic by some alternative must be the D-Topic. A declarative sentence with S-Topic and Focus thus allows for reconstructing a set of potential D-Topics. This set is called the Topic Value of $S, \llbracket S \rrbracket '$.

(9) a. Bill [ate ICE CREAM $]_{\mathrm{F}}=>$ What did Bill do?

b. $[\mathrm{BILL}]_{\mathrm{T}}$ [ate ICE CREAM $]_{\mathrm{F}}=>$ What did Bill do? or What did Bob do? or What did the boys do? or What did you do? or...

c. $\llbracket$ BILL $_{\mathrm{T}}$ [ate ICE CREAM $]_{\mathrm{F}} \rrbracket^{\prime}=\llbracket \lambda \mathrm{P} . \exists \mathrm{GQ} \in \mathrm{ALT}(\lambda \mathrm{R} \cdot \mathrm{R}(\mathrm{Bill})) \&$ $\mathrm{P}=\lambda$ p.. $\mathrm{V} \in \mathrm{ALT}$ (eat(icecream) \& $\mathrm{p}=\mathrm{GQ}(\mathrm{V}) \rrbracket^{\circ}$

We thus replace (6) by (10):

(10) Discourse Appropriateness Condition (final): 
For a question/answer pair Q/A to be wellformed, it must hold that $\llbracket Q \rrbracket^{\circ} \in \llbracket A \rrbracket^{\mathrm{t}}{ }^{2}$

According to this theory, sentences (1) through (3) contain Focus and - at least in the case of (1) and (2) - Topic accents. Both accents have a certain pragmatic function, i.e. they serve to make the utterance suited to a particular Discourse Topic. In turn, a single sentence with intonational structure defines the set of potential contexts (D-Topics) it can be used in.

\section{Strong Readings for Weak Determiners}

\subsection{Wide Focus}

Let us now try to apply our guessing strategy to (1), repeated here.

(11) a. [Some $]_{\mathrm{T}}$ cowboys decided [to stay home $]_{\mathrm{F}}$.

b. $\llbracket(11 . \mathrm{a}) \rrbracket^{\mathrm{t}}=\llbracket \lambda \mathrm{P} . \exists \mathrm{Q}[\mathrm{Q} \in \mathrm{ALT}$ (some) \& $\mathrm{P}=\lambda \mathrm{p} . \exists \mathrm{R}[\mathrm{R} \in \mathrm{ALT}(\mathrm{stay}($ home $)) \&$ $\mathrm{p}=\mathrm{Q}($ cowboys $)(\lambda \mathrm{x} \cdot \operatorname{decide}(\mathrm{x}, \mathrm{R}(\mathrm{x})))]]^{\mathrm{o}}$

In (11.b) I have given a formal-language equivalent to the Topic Value of (11.a). More perspicuously, what we do is replace both S-Topic and Focus by contextually relevant alternatives, here determiners and infinitival clauses.

(12) $\{$ the/all/many/some/two\} cowboys decided \{to stay home, to go gambling/to shave...

We know that the second pair of curly brackets - the Focus alternatives - reduces to something as simple as on what. As for the first - the S-Topic - there is no way to decide which alternative equals the one in the D-Topic (note that by (10) it suffices that any old element in $\llbracket(11 . a) \rrbracket^{\prime}$ equal the D-Topic). We thus conclude that the D-Topic must have been one of the questions in (13).

(13) What did the/all/most/some/two cowboys decide on?

Whichever of these (or similar) questions might have been the actual $\mathrm{D}$ (iscourse)Topic, it is about a group of cowboys. That is, we know upon hearing (11.a) even in isolation - that it requires a D-Topic that has to do with cowboys. Taking these to be a superset of the 'some cowboys' (a superset antecedent) in (11.a) gives us a partitive reading. One such case is illustrated in (14).

(14) There were twenty cowboys in the saloon. [TWO $]_{T}$ cowboys were watching the door.

In other words, the partitive effect in a sentence like (11.a) is not due to the semantics of the sentence itself, but to the kind of contexts it indicates. We infer that there must be an antecedent NP which shares the head noun with the subject in (11.a). Let us call this part-of relation between the NP and its antecedent a 
token partitive. Token partitivity is just one possibility: The antecedent of two cowboys might as well be a disjoined set of cowboys, i.e. a co-hyponym. This interpretation is the most plausible in a case like (15).

(15) The town was bursting with tension. A cowboy was standing next to the door of the saloon, nervously playing with his gun. $\mathrm{TWO}_{\mathrm{T}}$ cowboys were posted next to each window.

Two cowboys in (15) cannot be a subset of the one cowboy mentioned before (as in (14)). Nevertheless its intonational contour - the S-Topic accent - is clearly licensed by the earlier NP a cowboy, with which it shares the head noun (let us call this a token partitive). The treatment proposed here covers both these cases, since it has nothing to say about the relation between referents but only between sets of propositions. Nor does it require that the NPs involved are referential at all, which allows us to account for generic partitives as in (16).

(16) Q: What do you call a group of three musicians?

A: Dunno. FOUR $_{\mathrm{T}}$ musicians you call a QUARTET $\mathrm{F}$.

$\llbracket(16 \mathrm{~A}) \rrbracket^{\mathrm{f}}$ differs from $\llbracket(16 \mathrm{Q}) \rrbracket^{\circ}$ in much the same way as $\llbracket(8 . c) \rrbracket^{f}$ from $\llbracket(8) \rrbracket^{\circ}$ above: It answers a slightly different question, using the S-Topic to indicate that difference. Again, this is all the effect we ascribe to the intonational marking, regardless of the referential status of the NPs thus marked.

Let us briefly compare this treatment to others proposed in the literature. According to some authors, weak determiners are ambiguous between a cardinal and a quantificational version (Partee 1987, Diesing 1992, de Hoop 1992). The latter is represented as a generalized quantifier (the former is presumably just a cardinality predicate). Note that this does not prima facie explain the meaning difference. It only does if we make the additional assumption that the generalized quantifier presupposes something about the set that forms its restrictor. This is usually assumed to be an existential presupposition, e.g. to the effect that the restrictor set is presupposed to be non-empty. It should be noted, however, that this cannot be a presupposition in the usual sense, e.g. knowledge assumed to be shared by speaker and hearer, since examples like (17) clearly do not involve any existential commitments on the part of the person asking.

(17) Q: Are there any cookies in this room?

A: SOME $_{\mathrm{T}}$ cookies are [in the cupboard] $]_{\mathrm{F}}$.

Furthermore such theories do not explain why the strong determiners always bear a Topic accent. It has to be a lexical property of the quantifier version that it is obligatorily associated with a Topic accent, while its cardinal twin must be lexically excluded from being thus marked. Finally notice that the 'partitive' option is available both for existential and for generic NPs on the account advocated here (see (14) and (16) above). That means that the property of being 'partitive' cannot be tied to the property of being a true quantifier: both existentials and generics show the pertinent effect if marked as S-Topics. ${ }^{3}$

An approach towards these phenomena that is much closer to the present 
one is to assume that the partitive weak quantifiers do not establish a relation between sets but between discourse referents. A framework in which such an effect is derived compositionally is presented in Jäger 1995, according to which the NP TWO cowboys must introduce a discourse referent that is a subset of a referent already in the file. At first glance this analysis must surrender to examples of token partitivity like (15) above. But this can be fixed by appropriate meaning postulates that make available supersets and the like. Problems arise, however, with generics as well as with examples like (17). Obviously, we'd have to introduce additional machinery, e.g. discourse structures that allow for things like generic individuals and potential individuals.

\subsection{Narrow Focus}

Let us now turn to an argument against localizing the 'partitive' effect in the semantics of the determiner altogether. Consider (2.b), repeated here as (18.b).

(18) a. [THREE $]_{\mathrm{T}}$ boys [walked to the STATION $]_{\mathrm{F}}$.

b. $[\mathrm{THREE}]_{\mathrm{T}}$ boys $[\mathrm{WALKED}]_{\mathrm{F}}$ to the station.

Recall that according to the theory advocated here, the descriptive content of an antecedent NP is reconstructed from that part of the sentence that is neither Focus nor S-Topic (let us call this the Background). No reference was made to the notions of $\mathrm{N}$-argument and $\mathrm{V}$-argument in the sense of section 0 above. So far we only looked at examples parallel to (18.a), where in fact only the head noun of the quantified NP is in the Background. Here, using the Background to reconstruct the D-Topic comes down to the same as using the $\mathrm{N}$-complement of the determiner. However, if the Background contains more material than just the $\mathrm{N}$-argument, we predict that a similar strategy will apply to (non-focused) material from the V-argument. This is what happens in (18.b), where the accent on the verb unambiguously indicates narrow Focus. First, we infer again that there must have been an antecedent NP denoting boys, but furthermore, we take it that these boys all got to the station somehow (or at least tried to). ${ }^{4}$ That is, we find the very same effect as in the previous subsection, except that this time the reconstructed antecedent gets its descriptive content from material outside of the $\mathrm{N}$-argument. This clearly indicates that any theory that derives the partitive effect from just some requirement on the set denoted by the $\mathrm{N}$-argument falls short of capturing the phenomenon entirely.

\section{Strong Quantifiers}

In the previous section we only dealt with cases where the Focus effect wasn't truth-conditional but concerned the presupposition of the sentences. Therefore, the pragmatic account lent itself quite naturally to analyzing these cases. If we now want to go on to cases involving strong quantifiers, it seems we have to find some way to let Focus influence truth-conditions, for changes in truth conditions are exactly what we find. And in fact, we will do so by making use of 
Westerståhl's (1985) concept of Resource Domain Variables, i.e. free variables that function as additional restrictors on the quantifier, and whose value is provided anaphorically by the discourse (i.e. the assignment). In using RDVs rather than semantic rules I am making two essential claims: First, that TFBS does not directly enter the compositional semantics but only through the discourse, that is, uniformly and at few, well-defined places (this point is made e.g. in von Fintel 1994, and - to a certain extent - in Rooth 1992). And second, accordingly, that the Focus effect on quantifiers is less direct than assumed in the semantic approaches, i.e. that specific contexts can provide specific RDVs, which in turn yield truth conditions that are not available in the semantic approaches.

The effect of RDVs can most easily be seen in an example like (19)/(19.a): Of course, most women does not mean most of the women in the world, but most of the women standing in the market place. Using RDVs we express this by superscripting the quantifier with a variable - most ${ }^{C}$ - which represents a contextually specified variable (here: the property of being in the market place) that is intersected with the meaning of the $\mathrm{N}$-argument: \most $\rrbracket$ ( \women $\rrbracket \cap$ $\llbracket C \rrbracket)(\llbracket$ stood in the front $\rrbracket)$. Note that just as almost every sentence involving quantifiers like every, most etc. is likely to be false without RDVs, these very sentences would all trivially be true if RDVs were freely available (e.g. if $\mathbf{C}$ were systematically taken to equal the meaning of the $\mathrm{V}$-argument). Obviously RDVs have to be heavily restricted by context. However, they cannot be determined unambiguously (cf.(19.b)), nor are they always provided by discourse referents or groups previously mentioned (as can be seen in (19.c)).

(19) There were dozens of people in the market place...

a. Most ${ }^{C}$ WOMEN stood in the FRONT.

b. Fifteen women entered the stage. MOST ${ }^{\mathrm{C}}$ women waved their hands.

( $C=$ being in the market place or $C=$ being on the stage)

c. Let us next turn to the village Simmersbach. Most ${ }^{\mathrm{C}}$ men over 55 are unemployed.

$(C=$ being/living in Simmersbach $)$

What I will argue for is (of course) that TFBS helps us guessing contexts, and that context, in turn, helps us guessing RDVs.

Again, if nothing but the N-argument is in the Background - as in (20.a) no effects obtain in zero-context. All we learn about the D-Topic from a sentence like (20.a) is that boys figure in it, which, taken as the property assigned to $\mathbf{C}$, makes no difference wrt. truth-condition. Accordingly, we only notice the effect of RDVs with sentences that cannot possibly have wide Focus in the V-argument, such as (2.a), repeated here.

(20) a. $\left[\operatorname{most}^{\mathrm{c}}\right]_{\mathrm{T}}$ boys [walked to the STATION] $]_{\mathrm{F}}$.

b. $\left[\operatorname{MOST}^{\mathrm{C}}\right]_{\mathrm{T}}$ boys WALKED to the station.

c. $\llbracket$ most $\rrbracket(\llbracket$ boy $\rrbracket \cap \llbracket C \rrbracket)(\llbracket$ walk to the station $\rrbracket)$

$\lambda \mathrm{x} . \mathrm{x}$ is a boy who gets to the station somehow

In these cases we infer from the Background - '...boys ... to the station' - that 
boys headed towards the station must have been part of the previous discourse. If we restrict most - through the variable $\mathbf{C}$ - to the set of these boys, we obtain a truth conditional effect: the sentence seems to be about the proportion of walkers among those going to the station.

To appreciate this treatment and realize its advantages, let us see how a semantic account would handle these cases. Obviously, (20) boils down to something like 'take the V-argument minus the Focus and use it as an additional restrictor'. In Alternative Semantics there is a straightforward way to get something 'minus the focus', namely by forming the union set of the alternatives to that something. This is called trivializing a Focus value. For example while

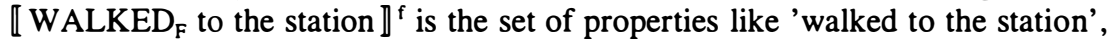
'drove to the station', or 'hitchhiked to the station', $U \llbracket \mathrm{WALKED}_{\mathrm{F}}$ to the station $\rrbracket^{\mathrm{f}}$ - its trivialization - is simply the property to have got to the station somehow. Thus, the semantic treatment can be summarized as in (21) (see appendix for details \& references).

(21) Restrict the quantifier by the trivialization of the focus value of the $\mathrm{V}$ argument $\llbracket \operatorname{most}(\mathrm{N})(\mathrm{V}) \rrbracket^{0}=\llbracket \operatorname{most} \rrbracket^{\circ}\left(\llbracket \mathrm{N} \rrbracket^{o} \cap \cup \llbracket V \rrbracket^{f}\right)\left(\llbracket V \rrbracket^{\circ}\right)$

Rule (21), too, interprets (20.b) as (20.c), as shown in (22).

(22) $\operatorname{most}\left(\llbracket\right.$ boys $\rrbracket^{\circ} \cap \cup \llbracket \mathrm{WALKED}_{\mathrm{F}}$ to the station $\left.\rrbracket^{f}\right)\left(\llbracket \mathrm{WALKED}_{\mathrm{F}}\right.$ to the station $\rrbracket^{0}$ )

The difference, however, is that (21) does so mechanically, while (20) is just one possible result of finding a decent RDV. Thus we should be able to construct specific contexts in which $\cup \llbracket \mathrm{V}$-argument $\rrbracket$ is not among the properties available as RDVs. And in fact, as Eckardt (1994) points out, in such cases the semantic account as in (21) leads to inappropriate truth conditions. Her example is given in (23).

(23) Max had to polish ten cars this afternoon. When I came back, six cars still stood in front of the garage, not even touched by Max. He had polished MOST $_{\mathrm{T}}$ cars CAREFULLY . $_{\text {. }}$

$\neq$ he had polished most of the cars that he polished carefully

Intuitively, (23) is plain nonsense: If Max hasn't even touched six out of ten cars, he cannot have polished most cars carefully. However, by (21) (23) should mean that most cars he polished, he polished carefully. If he had in fact polished three cars till they shone like the sun, (23) should be true. Thus, as Eckardt points out, (21) delivers wrong truth conditions. ${ }^{5}$

For the pragmatic account to handle this case we must assume that 'cars he polished' is not an available RDV in this specific context (while both 'the cars he ought to polish' and 'the cars he didn't polish' are, as can be seen by replacing the last sentence in (23) by He had smeared MOST cars with some undefinable substance). This seems plausible, given the fact that in general, differences between two given sets cannot antecede anaphors.

To sum up this section, I have shown that truth conditional effects with 
strong quantifiers can be accounted for pragmatically, if we make use of RDVs. A strong argument for this treatment can be made using examples like Eckardt's (23), where the pragmatic account - despite its general unspecificity - does not allow for the truth conditions assigned by the semantic one (and is right in doing so).

\section{Focus in the N-Argument}

Let us finally turn to cases like (3), repeated here:

(24) $\mathrm{Few} \mathrm{FEW}_{\mathrm{T}}$ INCOMPETENT $_{\mathrm{F}}$ cooks applied.

The example is taken from Herburger 1992, where cases like these are called focus-affected readings. Herburger makes the strong claim that these differ from parallel constructions without this accent pattern in terms of truth conditions. For this to hold, one has to assume that $f e w$ is an asymmetric quantifier, i.e. that $\operatorname{FEW}(\mathbf{A})(B)$ is not equal to $\mathbf{F E W ( B ) ( A ) . ~ T h e ~ r e a s o n ~ i s ~ t h a t ~ H e r b u r g e r ' s ~ a c c o u n t ~}$ - as all semantic accounts - conjoins sentence internal material with the restrictor and/or the nuclear scope. But when does that make a difference? If we assume that, in any event, the $\mathrm{N}$-argument is part of the restrictor and the $\mathrm{V}$-argument is part of the nuclear scope, adding $\mathrm{N}$ material to the restrictor (or $\mathrm{V}$ material to the nuclear scope) trivially won't make any difference. Adding $\mathrm{N}$-material to the nuclear scope makes no difference either, since all natural language determiners are conservative (i.e. $\mathrm{Q}(\mathrm{A})(\mathrm{B})$ equals $\mathrm{Q}(\mathrm{A})(\mathrm{A} \cap \mathrm{B})$. That leaves us with adding $\mathrm{V}$ material to the restrictor, which in turn only makes a difference if the determiner is non-symmetric (since otherwise $\mathrm{Q}(\mathrm{A})(\mathrm{B})=\mathrm{Q}(\mathrm{B})(\mathrm{A})=\mathrm{Q}(\mathrm{A} \cap \mathrm{B})(\mathrm{A} \cap \mathrm{B})$ and so forth). Therefore, truth conditional effects can only be expected with nonsymmetric quantifiers.

Let us now look at Herburger's argument. She seems to assume something along the lines of (25.a) to be the meaning of FEW. Then (25.b) correctly comes out as meaning 'only a small percentage of the marriages are marriages that are divorced in the first year'. But (25.c) will incorrectly mean 'only a small percentage of the incompetent cooks applied' ((25.d)).

(25) a. $\llbracket F E W(N)(V) \rrbracket \approx$ the cardinality of $\llbracket N \rrbracket \cap \llbracket V \rrbracket$ is small, compared to that of $\llbracket \mathrm{N} \rrbracket($ e.g. $|\llbracket N \rrbracket| /|\llbracket N \rrbracket \cap \llbracket V \rrbracket| \geq 5)$

b. Few marriages are divorced in the first year $(\approx$ less than $20 \%$ of the marriages are divorced in the first year)

c. Few INCOMPETENT cooks applied.

d. \# FEW(incompetent cooks)(applied) ( $\approx$ less than $20 \%$ of the incompetent cooks applied)

d. FEW(applying cooks)(incompetent) ( $\approx$ less than $20 \%$ of the applying cooks were incompetent)

e. $\quad \llbracket f e w(N)(V) \rrbracket=\llbracket F E W \rrbracket\left(U \llbracket N \rrbracket^{f} \cap \llbracket V P \rrbracket^{\circ}\right)\left(\llbracket N \rrbracket^{\circ}\right)$

But (25.c) can clearly be true, even if (almost) all incompetent cooks applied. What (25.c) seems to express is something like (25.d'): 'only a small percentage 
of the applying cooks were incompetent'. This meaning can be arrived at by using FEW only indirectly, as e.g. in (25.e) (this is not Herburger's analysis, though it is largely equivalent to it, see the appendix). In effect, (25.e) maps the Focus onto the nuclear scope and the Background onto the restrictor.

Note that the problem with (25.c) disappears under a symmetric analysis of few, such as $\llbracket$ few $(\mathrm{A})(\mathrm{B}) \rrbracket=1$ if $|\llbracket \mathrm{A} \rrbracket \cap \llbracket \mathrm{B} \rrbracket|<n$, where $n$ is some suitable low number. On such a purely cardinal reading ('the number of applying incompetent cooks is small'), (25.c) correctly describe the above scenario (all incompetent cooks applied) - admittedly alongside almost every other scenario, due to the complete vagueness of 'small', or more precisely, $n$.

Whether one prefers the strict semantic version or the (very) weak pragmatic one, thus, seems to be a matter of taste. Let me nevertheless mention a few points that $I$ take to argue in favor of the latter. First notice that few in (25.e) does not invoke any concept of 'smallness,' but only proportions. Thus (26.a) should be true (since the proportion of prime numbers among the natural numbers below is generally small), which seems wrong (since the total number of prime numbers smaller than a million is by no means small). By contrast, (26.b) is more likely to be judged correct, even though the proportion of prime numbers among the numbers smaller than ten is bigger than among those below a million.

(26) a. Few PRIME numbers are smaller than a million.

b. Few PRIME numbers are smaller than ten.

c. Many SWEDISH models were at my birthday party.

I think the point is even clearer in (26.c). Just like few, many seems to involve a contextually given measure of comparison, which in the case of many must be big, not just proportions. If only three models were at my birthday party, but two of them were Swedish, the proportion of Swedish among the models at my party is high (66\%), but nevertheless (26.c) would presumably be judged wrong. because two is simply not a big number on any count.

This account can be further refined if we assume an intensional (but symmetric) interpretation for few and many, roughly along the lines of (27) (cf. Keenan \& Stavi 1986, Fernando \& Kamp, this volume).

(27) $\llbracket \mathrm{FEW}(\mathrm{N})(\mathrm{V}) \rrbracket=$ the cardinality of $\llbracket \mathrm{N} \rrbracket \cap \llbracket \mathrm{V} \rrbracket$ is small, compared to what one might expect it to be

To arrive at Focus sensitive effects we superimpose on this a very simple 'rule' of Focus interpretation:

(28) BACKGROUND(FOCUS) $\leftrightarrow$ FOCUS, rather/to a higher degree than any of its alternatives, makes BACKGROUND(FOCUS) true

Applied to (25.c) we thus get something along the lines of (29).

(29) incompetent, rather than competent, makes it true that the number of such applying cooks is smaller than one might have expected 
Such an analysis also seems to fare better wrt. the classical Nobel prize example:

(30) Many SCANDINAVIANS won the Nobel prize.

a. asymmetric account: the number of Scandinavians among the nobel prize winners is big (say, more than $50 \%$ )

b. symmetric account: for Scandinavians (rather than Central Europeans) it holds that the number of such Nobel prize winners is bigger than one would expect it to be

(31) Few SCANDINAVIANS won the Nobel prize.

a. asymmetric account: the number of Scandinavians among the Nobel prize winners is below $20 \%$

b. symmetric account: for Scandinavians (rather than Central Europeans) it holds that the number of such Nobel prize winners is lower than one would expect it to be

In the real world, it is argued, Scandinavians won the Nobel prize quite often, not in absolute terms, but in terms of probability. Accordingly, (30) seems true, while (31) seems wrong, or at least unfair. This is the situation predicted by our symmetric analysis: (30.b) is true and (31.b) is false. On the asymmetric analysis things come out the other way around: (30.a) is clearly false while (31.a) is, I suspect, true. ${ }^{6}$

The last argument against a treatment along the lines of (25.e) is conceptual in nature, namely generality. It should be noted that focus-affected readings are systematically absent with strong quantifiers. Consider (32).

(32) Most INCOMPETENT cooks applied.

a. \# most cooks that applied were incompetent

b. 'incompetent' (rather than 'competent') characterizes that set $\mathrm{X}$ of cooks such that more than half of $\mathrm{X}$ applied

According to a rules analogous to (25.e), (32) should mean (32.a), which is clearly not the case. Note that what distinguishes this case from the ones discussed in the previous sections is that we do not only provide additional restrictor material, but are allowed to keep material from the $\mathrm{N}$-argument from occurring in the restrictor. Obviously, nothing like this happens in cases where truth conditions are unanimously detectable, which further strengthens our point that the mapping from syntax to semantics is first and foremost compositional and that the only source of deviance are additional restrictor variables. Note that the analysis sketched in this section seems to do pretty well on examples like (32), as shown by the paraphrase in (32.b).

\section{Conclusion}

To sum up, we have seen that the phenomena illustrated in (1) through (3) can be handled by the pragmatic account, and that often in specific contexts, the pragmatic account fares better in terms of truth and appropriateness conditions.

Partitive readings of weak quantifiers result from the fact that the noun, but not the quantifier, is part of the background. Hence an NP containing that 
noun must have been in the D-Topic. This also holds for cases of type partitives and generic partitives, not analyzable using a part-of analysis of 'partitivity' (section 2.1.).

Proportional readings of weak quantifiers are just a special case of partitives, namely one where the background is bigger than just the nominal argument to the quantifier (section 2.2).

Proportional readings of strong quantifiers result from the interplay of TFBS and the pragmatic process of finding a Resource Domain Variable. There is no direct association of the Focus value with the resource domain variable, which would yield inappropriate truth conditions (section 3 ).

Focus affected readings with few and many and NP internal Focus are again not due to direct association with Focus but should be handled using a symmetric, possibly intensional analysis and a general principle of Focus interpretation (section 4).

If something along these lines is correct, we seem to face a considerably simpler picture than we could expect. Not only can we dispense with additional transformations, lexical ambiguity, and specific semantic rules. We are also able to maintain a pretty restrictive theory of where and to which degree pragmatic factors such as FTBS can enter semantics proper. In particular, we can attribute to the FTBS a uniform effect on sentence meaning, an effect that does not rely on lexeme or construction specific assumptions.

What I haven't dealt with in this paper is the question why of all the possible ways to reconstruct a context for these sentences we chose those that yield the paraphrases given in (1) through (3), when we hear them out of context. Roughly, what we do in the default case is form a definite description D from the Background and interpret Q N as 'Q of the D' (this doesn't work literally for (3), due to the reasons discussed at the end of the last section). Furthermore we try to assume that the Focus is maximally big, i.e. we'd hardly interpret MOST boys walked to the STATION with narrow Focus on the PP, meaning 'most boys who walked somewhere, walked to the station', unless we are forced to do so by other contextual clues. Thus we usually only become aware of 'proportional' effects if Focus projection is blocked, e.g. if an adverbial or a verb is accented. A first guess would be that all this follows a strategy of 'minimal accommodation,' i.e. the attempt to reconstruct a maximally small context with minimal effort. This aspect, however, requires more study.

\section{Appendix: Differences and similarities - A look at four approaches}

In this appendix I'll review the works mentioned rather briefly in the text in some more detail. I try to focus on the differences and similarities between the approaches. Since all analyses are concerned with (a subset of) the four constructions discussed in the main text, I will use the following shorthands: SQ$\mathrm{FV}=$ strong quantifier with Focus in the V-argument; SQ-FN = strong quantifier with Focus in the N-argument; WQ-FV = weak quantifier with Focus in the $\mathrm{V}$-argument (e.g. three, some etc.); WQ-FN = weak quantifier with Focus 
in the $\mathrm{N}$-argument (i.e. few and many).

\subsection{Eckardt 1994}

Eckardt (1994) uses a rule of LF construal which basically equals that of Heim 1982: Quantified noun phrases are adjoined to the clause, followed by subextraction of the quantifier alone. Thus (33.a) is mapped to (33.b).

(33) a. MOST $\mathrm{T}_{\mathrm{T}}$ boys kissed Amalie PASSIONATELY $\mathrm{F}_{\mathrm{F}}$

b. [s $\operatorname{most}_{1}\left[s\left[{ }_{N P} t_{1} \text { boys }\right]_{2}\left[s t_{2}\right.\right.$ kissed Amalie PASSIONATELY] $]$

Most then is interpreted as taking the ordinary and the focus value of the sentence as its argument, that is, the distinction between $\mathrm{N}$-argument and $\mathrm{V}$-argument is irrelevant. To facilitate comparison I give an equivalent rule in (35).

(34) a. $\llbracket$ most $A \rrbracket=1$ iff there are more $x \in \llbracket A \rrbracket^{\circ}$ than $x \in \cup \llbracket A \rrbracket^{f}$ and $\notin \llbracket A \rrbracket^{\circ}$

b. The number of $\mathrm{x}$ which are boys and kissed Amalie passionately is bigger than the number of $\mathrm{x}$ which are boys and kissed Amalie some other way

(35) Rule E: $Q(N)(V)$ is interpreted as $Q\left(U \llbracket N \rrbracket^{f} \cap \cup \llbracket V \rrbracket^{f}\right)\left(\llbracket N \rrbracket^{\circ} \cap\right.$ IV $]^{\circ}$ )

For SQ-FV (as well as WQ-FV) Rule E equals the semantic accounts discussed in sections 2 and 3 , that is, it derives the default interpretation but fails to account for cases like (23) above. For SQ-FN and WQ-FN, Rule E is equivalent to (25.e) discussed in section 4 , that is, it can treat weak quantifiers but fails with strong ones. Thus (36.a) becomes (36.b) at LF and ultimately means (36.c).

(36) a. $\operatorname{MOST}_{\mathrm{T}} \mathrm{RED}_{\mathrm{F}}$ balloons burst.

b. $\operatorname{most}_{1}\left[{ }_{s}\left[_{\mathrm{NP}} t_{1} \text { RED balloons }\right]_{2}\left[{ }_{\mathrm{s}} t_{2}\right.\right.$ burst $]$

c. Most balloons that burst were red.

\subsection{Geilfuß 1993}

Geilfuß (1993) avoids subextraction of the determiner by using Rooth's (1992) squiggle operator. Thus (33.a) is represented as (37). This treatment is equivalent to Rule $G$ in (38).

(37) $\left[{ }_{s}\left[\mathrm{NP} \operatorname{most}\left(\mathrm{C}_{7}\right) \text { boys }\right]_{1}\left[{ }_{s}\left[_{s} t_{l}\right.\right.\right.$ kissed Amalie PASSIONATELY] $\left.\left.\mathrm{C}_{7}\right]\right]$

(38) Rule G: $Q(N)(V)$ is interpreted as $Q\left(\llbracket N \rrbracket^{\circ} \cap \llbracket V P \rrbracket^{f}\right)\left(\llbracket V P \rrbracket^{\circ}\right)$

For SQ-FV and WQ-FV, Rule E and Rule G are equivalent (hence equally deficient). For SQ-FN the outcome depends on where $\sim \mathrm{C}_{7}$ is adjoined. If it is adjoined to the $\mathrm{N}$-argument, the Focus effect is wiped out (since $\cup \llbracket N \rrbracket^{\mathrm{f}} \supseteq$ $\llbracket N \rrbracket^{\circ}$ ). If it is adjoined to the $\mathrm{V}$-argument, the sentence becomes a tautology, since $U \llbracket \mathrm{V} \rrbracket^{\mathrm{f}}=\llbracket \mathrm{V} \rrbracket^{\mathrm{o}}$; the latter case is given in (39).

(39) a. [s $\left[{ }_{N P} \operatorname{most}\left(C_{7}\right) \text { RED balloons }\right]_{2}\left[{ }_{s}\left[s_{2}\right.\right.$ burst $\left.\left.] \sim \mathrm{C}_{7}\right]\right]$

b. $\quad \operatorname{most}\left(\llbracket\right.$ red balloons $\rrbracket^{\circ} \cap \cup \llbracket$ burst $\left.\rrbracket^{\top}\right)\left(\llbracket\right.$ burst $\left.\rrbracket^{\circ}\right)$ 
Since $\cup \llbracket$ burst $\rrbracket^{\mathrm{f}}=\llbracket$ burst $\rrbracket^{0},(39 . \mathrm{b})$ is a tautology.

\subsection{Herburger 1992}

Herburger's treatment differs from the one discussed in section 4 in that she uses syntactic movement to construct the relevant configuration: 'a focused predicate inside a VP-internal NP extraposes at LF. Thereby it ... becomes the main predicate' (Herburger 1992:13). Thus, an LF roughly as in (40.a) is obtained, which is interpreted as in (40.b).

(40) a. [s $e$ apply [vp[vp [NP few cooks] $t_{V}$ ] incompetent]

b. $\exists e\left[\operatorname{apply}(e) \& \mathrm{few}_{\mathrm{x}}(\operatorname{cook}(\mathrm{x}, \mathrm{e}))(\Theta(\mathrm{x}, \mathrm{e}) \&\right.$ incompetent $(\mathrm{x}))$

This analysis fails for non-intersective adjectives:

(41) Few ALLEGED/WOULD-BE/FORMER cooks applied ( $\neq$ few of the applying cooks were alleged/would-be/former cooks)

We can repair this by transforming the treatment into the almost equivalent semantic treatment (25.e), repeated here in a slightly different form:

(42) Rule H: [Q N'] VP is interpreted as $\llbracket Q \rrbracket\left(U \llbracket N^{\prime} \rrbracket^{f} \cap\right.$

$$
\left.\llbracket \mathrm{VP} \rrbracket^{\circ}\right)\left(\llbracket[\ldots]_{F} \rrbracket^{\circ}\right)=\llbracket Q \rrbracket\left(\cup \llbracket N^{\prime} \rrbracket^{f} \cap \llbracket V P \rrbracket^{o}\right)\left(\llbracket N^{\prime} \rrbracket^{o}\right)
$$

This rule handles (41) correctly. In fact it is equivalent to Rule E for all four cases. It should be noted though that Herburger does not deal with SQ-FV or WQ-FV. The problem with SQ-FN is overcome by a syntactic constraint against extraposing from strong NPs. That, however, would presumably also block the derivation of SQ-FV. Furthermore, such a constraint cannot be used in the semanticized version needed to handle (41).

\subsection{De Hoop 1995 and de Hoop \& Solà 1995}

De Hoop and Solà assume that Focus effects only occur with Focus in the Vargument. Thus WQ-FN is treated as non-truth conditional (an analysis we have adopted in section 4), and no problems with SQ-FN arise. This is obtained through a rule like (43).

(43) Rule HS:

$\mathrm{Q}(\mathrm{N})(\mathrm{V})$ is interpreted as $\mathrm{Q}\left(\llbracket \mathrm{N} \rrbracket^{\circ} \cap \mathrm{X}\right)\left(\llbracket \mathrm{VP} \rrbracket^{\circ}\right)$,

where $X$ equals $\cup \llbracket N \rrbracket^{f}$ if the Focus is within $N$, or $X$ equals $\cup \llbracket V \rrbracket^{f}$ if the Focus is within $\mathrm{V}$ (simplified, see below)

SQ-FV and WQ-FV are treated equivalently to Rules E, G, and H ((44.a)). SQFN and WQ-FN do not receive any special treatment: Any effect of focus in the first argument is wiped out ((44.b)).

(44) a. Most/few cats purred LOUDLY. $\Rightarrow$ most/few cats that purred purred loudly (truth-conditional effect)

b. Few SWEDISH cats purred. $\Rightarrow$ few Swedish cats purred loudly (no 
truth-conditional effect)

In de Hoop 1995 a more sophisticated rule than (43) is presented, namely one that states $(45)$.

(45) The Focus value of the focus-containing constituent ( $\mathrm{X}$ in the sense of (43)) is intersected with the property the quantifier lives on.

(45) differs from (43) only for quantifiers that live on their second argument. According to de Hoop 1995 this holds for the quantifier only, as the equivalence in (46.a) shows.

(46) a. $\llbracket \mathrm{ONLY}(\mathrm{N})(\mathrm{V}) \rrbracket=\llbracket \mathrm{ALL}(\mathrm{V})(\mathrm{N}) \rrbracket=\left\{\mathrm{w}: \llbracket \mathrm{V} \rrbracket_{\mathrm{w}} \subseteq \llbracket \mathrm{N} \rrbracket_{\mathrm{w}}\right\}$

b. Only cool people drink beer $\equiv$ All beer drinkers are cool people $\equiv$ the beer drinkers are a subset of the cool people

c. Only SWISS linguists drink.

$\neq$ All drinkers are Swiss linguists.

$=$ All drinking linguists are Swiss.

d. $\quad$ only $(\mathrm{N})(\mathrm{V}) \rrbracket^{\circ}=\llbracket$ ONLY $\rrbracket\left(\llbracket N \rrbracket^{\circ}\right)\left(U \llbracket N \rrbracket^{\mathrm{f}} \cap \llbracket \mathrm{V} \rrbracket^{\circ}\right)(=$ $\left.A L L\left(U \llbracket N \rrbracket^{\mathfrak{f}} \cap \llbracket V \rrbracket^{\circ}\right)\left(\llbracket N \rrbracket^{\circ}\right)\right)$

Cases of SQ-FN such as (46.c) now require a modification of truth conditions. (43) and (45) jointly cause (46.c) to be interpreted as in (46.d).

However, one can plausibly argue that only in (46.b) and (46.c) is not a determiner, but an NP modifier (cf. Rooth 1985). At least in cases like (47) which show the same truth conditional effects as (46.c) - this is clearly the right analysis.

(47) Only the/some/few/two SWISS linguists drink excessively.

Analyzing only SWISS linguists as [NP only [NP SWISS linguists]] we can derive the correct interpretation without additional rules by the usual crosscategorial semantics of only as given in Rooth 1985.

(48) Only [NP SWISS $_{\mathrm{F}}$ linguists] drink.

a. 【SWISS linguists $\rrbracket^{\circ}=\lambda \mathrm{P}_{\mathrm{et}} \cdot \exists \mathrm{X}[\operatorname{swiss}(\mathrm{X}) \&$ linguist $(\mathrm{X}) \& \mathrm{P}(\mathrm{X}) \rrbracket \approx$ the set of properties had by one or more Swiss linguists

b. 【SWISS linguists $\rrbracket^{\prime}=\lambda R . \exists S \in A L T$ (swiss) \& $R=\lambda P_{4 t} . \exists X[S(X) \&$ linguist $(X) \& P(X)] \approx$ the set of sets of properties $P$ such that there is a nation and one or more linguists from that nation have that property

c. $U \llbracket$ SWISS linguists $\rrbracket^{f} \approx$ the set of properties $P$ such that there are one or more linguists who have that property $P$

d. $\llbracket$ only SWISS linguists $\rrbracket^{\circ}=\lambda P . \forall S \in A L T$ (swiss) $[\exists X[S(X) \&$ linguist $(X) \& P(X)] \rightarrow S=$ swiss] $\approx$ the set of properties $P$ such that if there are one or more linguists with that property, they are Swiss

e. 【[only SWISS linguists] drink $\rrbracket^{\circ} \approx$ drinking is a property such that if any linguist has it, he is Swiss $\approx$ all drinking linguists are Swiss (cf.(46.c))

Thus we can avoid using (43) and (45). 


\section{Endnotes}

1. In addition we need a condition to ensure that the D-Topic DT itself may only provide information that is contextually given, i.e. part of the Common Ground CG (e.g. it must hold that $\cup \llbracket \mathrm{DT} \rrbracket \supseteq \llbracket C G \rrbracket)$. In tandem these two conditions ensure that for every sentence $S, \cup \llbracket S \rrbracket^{f} \supseteq C G$, i.e. the Background of $S$ is uninformative (see Büring 1995, chap. 2 and Büring t.a.).

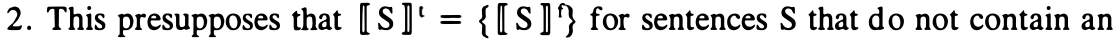
S-Topic. This treatment is a straightforward extension of Rooth 1985.

3 . Note that the analysis proposed here does not need to make any commitment as to the nature of the generic/ existential contrast. We only note that both types of indefinites become 'partitive' if marked by a topic accent. Strong indefinites are just Topic marked existentials.

4. One often gets the impression that the other boys' getting to the station in some alternative way is entailed or presupposed by a sentence like (18.b), i.e. that it must be continued by something like '...the others stayed at home' or '...the others took a cab'. On closer inspection, however, this turns out to be just a conversational implicature. It follows in cases with superset antecedents like 'the boys that got to the station somehow' by conversational implicature due to the Gricean Maxim of Quantity: If the speaker knew that all boys walked, she shouldn't use (18.b), which is less informative than possible. This implicature can vanish just as the superset antecedent can (cf. (17) above):

(i) I was curious whether the boys would make it to the station. Well, $\mathrm{TWO}_{\mathrm{T}}$ boys WALKED $\mathrm{W}_{\mathrm{F}}$ to the station.

...the others didn't get there at all/...I don't know about the others.

5. Eckardt (1994) considers the possibility of requiring that, on top of (21) (which in effect means: instead of it) it must hold that $\llbracket N \rrbracket \subseteq U \llbracket V \rrbracket^{f}$. That is, it is entailed (or maybe presupposed) that, say, in Max polished MOST cars CAREFULLY he polished all cars to begin with. Accordingly (23) would be plainly contradictory. This, however, is too strong, cf. (i):

(i) Max polished MOST $_{T}$ cars CAREFULLY , but I am not sure whether he polished all of them.

All we find here is that the speaker must not know that Max polished all cars carefully (due to Gricean reasoning again), which includes the possibilities of him polishing the others i) carelessly, ii) in some unknown manner, or iii) not at all. See also note 4 above.

6. This argument goes through even without assuming an intensional analysis. The important thing here is that (25.e) compares the number of Scandinavian winners to the total number of non-Scandinavian winners, while (28) compares it to the number of winners from each other region. Thus five Scandinavians would count as many even compared to 100 non-Scandinavians, provided no single alternative (say the US) has more than five. The intensional interpretation, on top of that, allows for five Scandinavians to weigh more than 10 Americans, say, due to the total number of applicants from both countries. 


\section{References}

Büring, D. (1995) The 59th Street Bridge Accent. PhD dissertation. Tübingen. Büring, D. (t.a.) Topic. In: Bosch, P. \& R. van der Sandt (eds) The Focus Book. Diesing, M. (1992) Indefinites. Cambridge, MA: MIT Press.

Eckardt, R. (1994) Adverbs in Focus. Ms. Stuttgart University.

von Fintel, K. (1994) Restrictions on Quantifier Domains. PhD dissertation, University of Massachusetts at Amherst.

Geilfuß, J. (1993) Nominal Quantifiers and Association with Focus. In: Ackema, P. \& M. Schoorlemmer (eds) Proceedings of the Workshop on the Semantic and Syntactic Analysis of Focus. Utrecht: OTS. 33-41.

Heim, I. (1982) The Semantics of Definite and Indefinite Noun Phrases. PhD dissertation, University of Massachusetts at Amherst.

Herburger, E. (1992) Focus and the LF of NP Quantification. In: Proceedings of SALT III.

Hamblin, C.L. (1973) Questions in Montague Grammar. Foundations of Language 10. 41-53.

de Hoop, H. (1992) Case Configuration and Noun Phrase Interpretation. PhD dissertation, Rijksuniversiteit Groningen.

de Hoop, H. (1995) Only a Matter of Context? Ms. Groningen.

de Hoop, H. \& J. Solà (1995) Determiners, Context Sets, and Focus. Talk presented at WCCFL 14. USC.

Jackendoff, R. (1972) Semantic Interpretation in Generative Grammar. Cambridge, MA: MIT Press.

Jäger, G. (1995) Topics in Dynamic Semantics. PhD dissertation. Berlin.

Keenan, E. \& J. Stavi (1986) A semantic characterization of natural language determiners. Linguistics and Philosophy 9.

Partee, B. (1987) Noun Phrase Interpretation and Type Shifting Principles. In: Groenendijk, J. \& D. de Jong \& M. Stokhof (eds) Studies in Discourse Representation Theory and the Theory of Generalized Quantifiers. Dordrecht: Foris.

Partee, B. (1989) Many quantifiers. In: Powers, J. \& K. de Jong (eds) Proceedings of the fifth Eastern States Conference in Linguistics. Ohio State University.

Rooth, M. (1985) Association with Focus. PhD dissertation. University of Massachusetts at Amherst.

Rooth, M. (1992): A Theory of Focus Interpretation. Natural Language Semantics 1, 75-116.

Westerståhl, D. (1985) Logical Constants in Quantifier Languages. Linguistics \& Philosophy 8. 387-413. 